Gefässchirurgie 2022 27:129-134 https://doi.org/10.1007/s00772-021-00854-1

Angenommen: 29. November 2021 Online publiziert: 20. Dezember 2021

(c) Der/die Autor(en) 2021

\section{Physikalische Interventionen als Alternative/Ergänzung zum chirurgischen Wunddebridement}

\author{
Ultraschall, Kaltplasma und LASER/Photobiomodulation
}

\author{
Ursula Elisabeth Maria Werra ${ }^{1} \cdot$ Max Zinser $^{2} \cdot$ Bernhard Dorweiler' \\ ' Klinik für Gefäßchirurgie, Vaskuläre und endovaskuläre Chirurgie, Uniklinik Köln, Köln, Deutschland \\ ${ }^{2}$ Klinik für Plastische, Rekonstruktive und Ästhetische Chirurgie, Uniklinik Köln, Köln, Deutschland
}

Hintergrund: Die Versorgung von Patienten mit chronischen Wunden verursacht in Deutschland jährliche Kosten in Höhe von 8 Mrd. $€$.

Häufig ist ein chirurgisches Debridement notwendig. Wenn jedoch kritische Strukturen lokal angrenzen oder der Patient nur eingeschränkt narkosefähig ist, können die Einsatzmöglichkeiten limitiert sein.

Zusätzlich ist aus wirtschaftlichen Gründen eine mögliche Delegierbarkeit immer von Interesse.

Fragestellung: Wir präsentieren alternative sowie ergänzende Methoden zum chirurgischen Debridement aus dem Portfolio der physikalischen Interventionen. Material und Methoden: Ultraschallassistiertes Wunddebridement (UAW), Kaltplasma sowie Photobiomodulation basieren auf interessanten Grundprinzipien mit vielversprechendem Entwicklungspotenzial. Der Wirkmechanismus, die Evidenzlage sowie wirtschaftliche Aspekte werden vorgestellt.

Ergebnisse: Es zeigt sich insgesamt aufgrund der Inhomogenität der Daten eine nur schwache Evidenzlage. Auch einige Aspekte der Behandlungsmodalitäten sind noch nicht abschließend geklärt.

Das UAW stellt eine echte Alternative dar. Es erfährt eine hohe Patientenakzeptanz und ist ambulant durchführbar.

Die Wirksamkeit von Kaltplasma oder Photobiomodulation ist noch nicht abschließend beurteilbar. Es zeigen sich jedoch positive klinische Ergebnisse.

Diskussion: Das chirurgische Debridement ist auch aktuell (noch) nicht vollständig ersetzbar. Alternative Methoden wie das UAW können jedoch einen zusätzlichen Benefit bieten. Wunden können so teilweise frühzeitig (mit entsprechender Kostenersparnis) ambulant behandelt werden. Eine weitere wichtige Stärke stellt zudem die Wirksamkeit bezüglich multiresistenter Erreger dar.

Es besteht die Notwendigkeit zur Initiierung weiterer klinischer Studien, da die aktuelle Datenlage noch viele offene Fragen aufweist.

\title{
Schlüsselwörter
}

Moderne Wundtherapie · Photobiomodulation - Ultraschallassistiertes Debridement · Plasma . Chronische Wunden

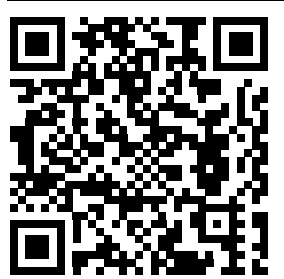

QR-Code scannen \& Beitrag online lesen

\section{Hinführung zum Thema}

In Deutschland gibt es geschätzt 1,7 Mio. Patienten mit chronischen Wunden. Aktuell kostet die Versorgung dieser Patienten 8 Mrd. $€$ pro Jahr $[1,10]$.

Häufig stellt sich die Notwendigkeit eines chirurgischen Debridements. In man- chen Fällen ist der Einsatz jedoch limitiert, sodass physikalische Methoden eine Alternative/Ergänzung darstellen können.

Wir stellen drei Verfahren vor, die in den letzten Jahren viel Aufmerksamkeit erhielten. Alle basieren auf interessanten Grundprinzipien mit großem Entwicklungspo- 


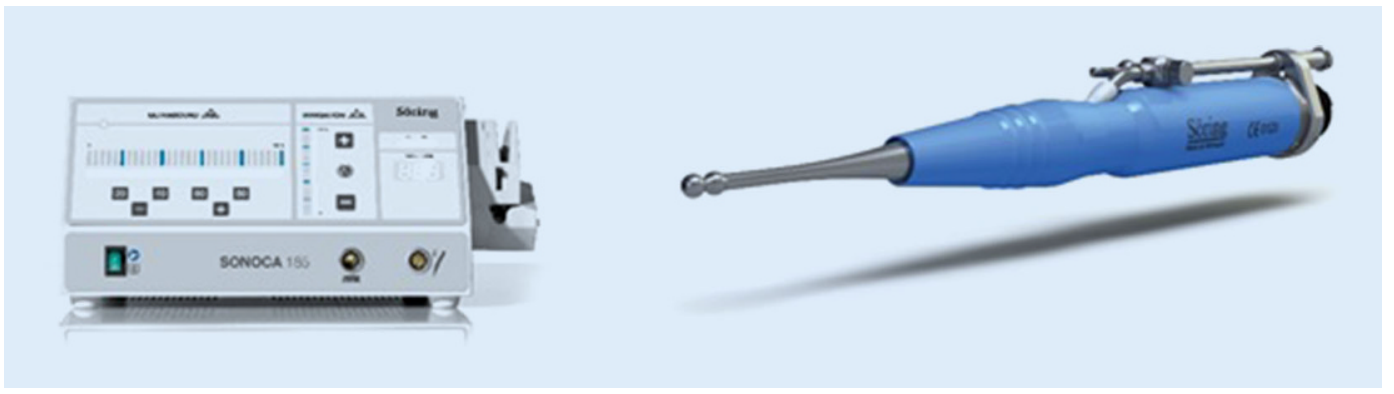

Abb. 14 Ultraschallgenerator und Ultraschallassistiertes-Wunddebridement(UAW)Instrument, mit freundlicher Genehmigung der Söring GmbH, Quickborn

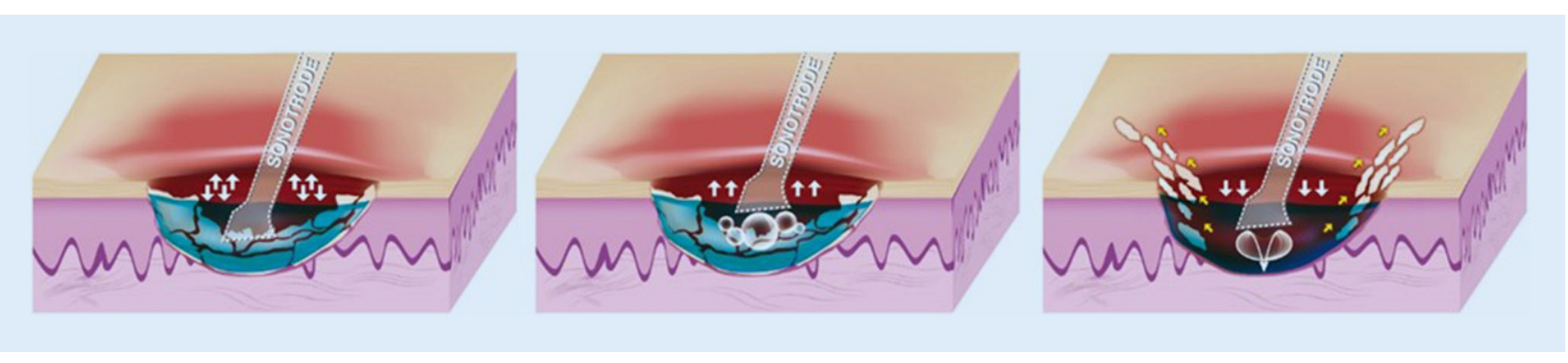

Abb. 2 ム Wirkprinzip des UAW (ultraschallassistiertes Wunddebridement) (vereinfachte Darstellung), mit freundlicher Genehmigung der Söring GmbH, Quickborn

tenzial. Wir diskutieren die Evidenzlage, aber auch wirtschaftliche Aspekte.

\section{Das chirurgische Wund- debridement}

Bei der Behandlung einer chronischen Wunde besteht meist die Indikation zum chirurgischen Debridement. Hierbei werden Beläge oder Nekrosen entfernt und Wundtaschen gesäubert, um eine Wundheilung zu ermöglichen. Dies geschieht definitionsgemäß bis in intakte anatomische Strukturen hinein. Insbesondere bei großflächigen Nekrosen, lokalen Entzündungszeichen, dem Vorhandensein von Fremdkörpern oder einer systemischen Infektion steht ein chirurgisches Debridement im Vordergrund [4, 9, 22].

Antikoagulanzien, Blutgerinnungsstörungen, eine eingeschränkte Narkosefähigkeit, aber auch die Nähe zu kritischen Strukturen oder eine nicht verbesserbare eingeschränkte Durchblutung können die Einsatzmöglichkeiten des chirurgischen Debridements limitieren [4, 9, 27].

\section{Sozialmedizinische und wirtschaftliche Faktoren}

Gerade bei älteren Patienten ist eine ambulante Therapie, wenn möglich, anzustreben. So erleiden etwa $30-50 \%$ der geri- atrischen Patienten im Krankenhaus ein Delir. Dies kann zu langfristigen kognitiven Dysfunktionen führen und erhöht die Letalität [30].

Es stellt sich zudem aus wirtschaftlichen Gründen die Frage der Delegierbarkeit von Maßnahmen. Das scharfe chirurgische Debridement ist an den Arzt/die Ärztin gebunden [24].

Die hier vorgestellten Anwendungen gelten als Wundbehandlung und erlauben nach entsprechender Schulung eine Delegation an nichtärztliches Personal.

\section{Ultraschallassistiertes Wund- debridement (UAW)}

\section{Wirkprinzip}

Das ultraschallassistierte Debridement (UAW) beruht auf dem Kavitationseffekt. Prinzipiell kann hier hochfrequenter Ultraschall $(<10 \mathrm{MHz})$ sowie niederfrequenter (20-60 kHz) Ultraschall eingesetzt werden. Mit einer Sonotrode werden zunächst Kavitationsbläschen erzeugt, welche dann durch die nächste Annäherung zum Implodieren gebracht werden. Dadurch entstehen "Microjets", die zum Debridement der Wunde führen. Das gesunde Gewebe wird dabei aufgrund des höheren Elastingehaltes nicht beeinträchtigt. Die Wunde wird kontinuierlich mit einer Flüssigkeit (z. B. NaCl) gespült. Entstehende Aerosole werden abgesaugt (- Abb. 1 und 2). Eine lokale Anästhesie der Wunde ist teilweise vonnöten.

Neben den mechanischen Aspekten (Nekrosektomie, Aufbrechen von Biofilmen) werden auch Prozesse auf zellulärer Ebene beeinflusst: Zellaktivität, Proteinsynthese und Fibrinolyse werden erhöht. Zusätzlich gibt es Hinweise darauf, dass u.a. Angiogenese und die Reißfestigkeit der Wunde positiv beeinflusst werden [7, 19, 29].

\section{Wirksamkeit/Evidenz}

Generell findet sich eine nur schwache Evidenzlage. Eine Cochrane-Analyse von Cullum et al. (2017) bezüglich der Anwendung bei Ulcus cruris venosum ergab mit Ausnahme einer qualitativ hochwertigen Studie keine belastbaren Daten, sodass keine Aussage getroffen werden konnte [8].

Nur das systematische Review von Chang et al. (2017) konnte 25 Studien bezüglich der Applikation von niederfrequentem Ultraschall einschließen, worin zumindest zwei Evidenzlevel-1- und drei Evidenzlevel-2-Studien enthalten waren [7].

Tierversuche zeigten eine erhöhte Fibrinolyse, Proteinsynthese sowie Angiogenese und verbesserte Reißfestigkeit der 


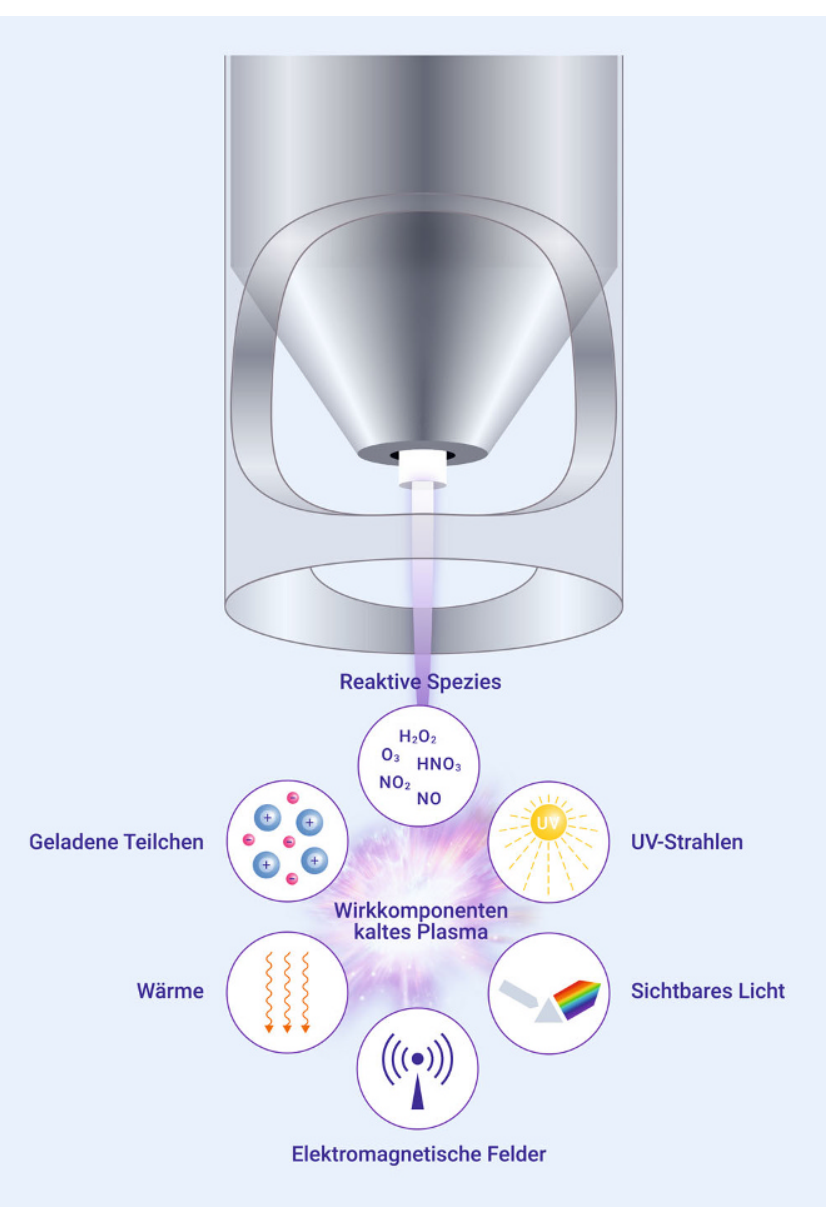

\section{Schlussfolgerung}

Das UAW stellt eine sinnvolle Ergänzung des Portfolios in der Behandlung chronischer Wunden dar. Es ermöglicht ein schonendes Debridement in der Nähe kritischer Strukturen und wird auch bei reduzierter Perfusionssituation empfohlen. Es kann ambulant durchgeführt werden und ist nicht an den Arzt gebunden. Die Aerosolbildung ist als kritischer Aspekt zu betrachten, sodass sicherlich $u$. a. das Tragen einer geeigneten Schutzkleidung empfehlenswert wäre. Die Delegierbarkeit macht das Verfahren wirtschaftlich attraktiv und es erfährt eine hohe Patientenakzeptanz. Hochwertige klinische Studien sollten die Methode weiter evaluieren.

\section{Kaltes atmosphärisches Plasma}

\section{Wirkprinzip}

Abb. $3<$ Kaltplasma: Prinzip, mit freundlicher Genehmigung der neoplas med $\mathrm{GmbH}$ Greifswald
Wunden. Auch die Expression inflammatorischer Zytokine wird reduziert. Hier fand sich eine signifikante Korrelation zwischen Reduktion der Wundgröße und TNF-alpha sowie Interleukin 1, 6, 8 und 11 [6]. Neben dem Debridement besteht der Hauptaspekt des UAW in der Reduktion des Biofilms sowie der Bakterienlast, auch bei multiresistenten sowie ausgeprägt biofilmbildenden Bakterien. Hier zeigt es sich teils dem chirurgischen Debridement überlegen $[7,16,17]$.

Amini et al. (2013) und Lázaro-Martínez et al. (2020) konnten bezüglich der Behandlung von Patienten mit diabetischem Fußsyndrom einen signifikanten positiven Einfluss einer Behandlung mit niederfrequentem Ultraschall auf die Wundgröße und Abheilungszeit - auch im Vergleich zum chirurgischen Debridement - nachweisen. Es zeigt sich zudem eine Reduktion des Exsudates sowie des Schmerzempfindens der Patienten, was für die Lebensqualität der Patienten einen großen Unterschied darstellen kann $[2,7,17]$.

\section{Wirtschaftlichkeit}

Firmen wie die Söring $\mathrm{GmbH}$ bieten seit einigen Jahren entsprechende kompakte Handgeräte an.

Das empfohlene Behandlungsregime beinhaltet drei Anwendungen pro Woche. Diese können ambulant, sowohl durch ärztliches als auch nichtärztliches Personal, durchgeführt werden.

Wie das chirurgischen Debridement kann auch UAW nach OPS 5-896 abgerechnet werden. Hierfür muss jedoch eine lokale Infiltrationsanästhesie vorgenommen werden oder eine neurologisch bedingte Analgesie vorliegen (z. B. bei DFS). Ansonsten fällt das UAW unter die OPS 8-192. Insbesondere durch die Delegierbarkeit sowie die Anwendung außerhalb eines OP-Saals besteht hier Potenzial zur Kostenreduktion [12].

Zudem erhöht sich die Wirtschaftlichkeit der Wundbehandlung an sich (seltenere Verbandswechsel durch Exsudatreduktion, weniger Schmerzmittelgabe).
Zur Erzeugung von Plasma werden Edelgase (z. B. Argon) in einem elektromagnetischen Feld angeregt und aktiviert (partiell ionisiert). Plasma besteht neben dem sichtbaren Licht aus elektromagnetischer Strahlung, UV-Strahlung $(U V B+C)$, lonen, Elektronen und freien Radikalenwie u.a. $\mathrm{O}_{3}, \mathrm{NO}, \mathrm{NO}_{2}$ (RONS). Durch die nur partielle lonisierung wird die geringe Temperatur von $30-40{ }^{\circ} \mathrm{C}$ generiert. Mit dem Plasmastrahl wird die Wunde schrittweise behandelt, indem der Applikator (z.B. kINPen/neoplas med, Medizinprodukt Ila) (mit Abstandshalter) mit einer Geschwindigkeit von circa $5 \mathrm{~mm} / \mathrm{s}$ über alle Areale der Wunde geführt wird. Das Verfahren ist schmerzfrei (-Abb. 3, 4 und 5).

Das Wirkprinzip ist noch nicht vollständig verstanden, beruht jedoch wohl hauptsächlich auf der Bildung der RONS, die z.B. die Bakterienwände schädigen können oder direkt mit Keratinozyten, Epithelzellen, Endothelzellen und Immunzellen selbst interagieren (z. B. im Bereich Lipidund Proteinstrukturen oder redoxabhängigen intrazellulären Signalkaskaden) [3, $6,11,13,14,20]$.

\section{Wirksamkeit/Evidenz}

Es liegen nur wenige, schwer miteinander vergleichbare Daten vor, da es viele unterschiedliche Plasmaquellen/ 


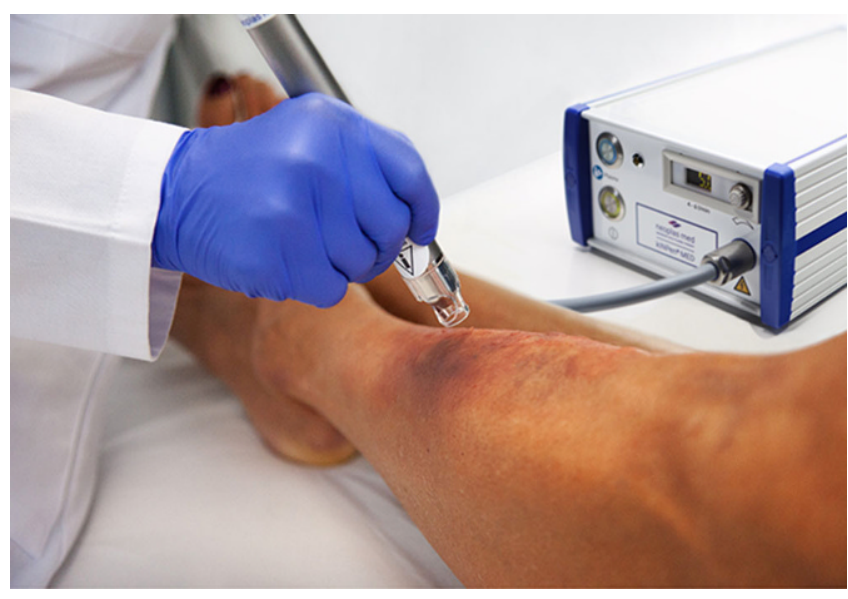

Abb. $4 \triangleleft$ Kaltplasma: Anwendung, mit freundlicher Genehmigung der neoplas med $\mathrm{GmbH}$, Greifswald
Erzeugungsverfahren gibt und die Effekte auch von Dosis und Anwendungszeit abhängen [11].

In vitro konnten neben einer erhöhten Freisetzung von Wachstumsfaktoren wie FGF-2 oder VEGF auch Veränderungen der Rezeptorstruktur der Zellen (insbesondere der Integrinrezeptoren) sowie eine erhöhte Expression von Genen wie Typ-1-Kollagen gezeigt werden [11, 18].

Insbesondere Patienten mit chronisch venösen Ulzera oder diabetischem Fußsyndrom scheinen von der Methode zu profitieren. Hier konnte in einzelnen Arbeiten eine signifikante Reduktion der Bakterienlast (unabhängig von Resistenzlage oder Biofilmbildung), Wundgröße und Schmerzintensität festgestellt werden [6, 14, 18, 20, 26].

Assadian et al. konnten 2019 in einem systematisches Review 13 Studien einschließen. Hier zeigte sich kein Vorteil bezüglich der bakteriellen Besiedlung oder Reduktion der Wundgröße. Die Autoren kritisierten die schlechte Datenqualität und empfahlen weitere Studien [3].

Interessanterweise zeigte eine Pilotstudie des Uniklinikums Essen aus dem Jahr 2020 Unterschiede bezüglich der Wirksamkeit (Wundgröße, Bakterienlast) je nach Applikationsfrequenz. Auch wenn sich prinzipiell ein signifikanter Vorteil gegenüber der Placebogruppe zeigte, war eine einmalige einer dreimaligen Anwendung pro Woche deutlich überlegen. Die Autoren diskutieren hier die potenziell schädliche Wirkung von RONS und UV-Strahlung bei höherer Applikationsfrequenz. Beim Schmerzempfinden wiederum war - wenn auch bei bei- den Gruppen signifikant gebessert - die höhere Applikationsfrequenz überlegen [20].

\section{Wirtschaftlichkeit}

Die Behandlung mit Kaltplasma wird aktuell noch nicht von der gesetzlichen Krankenkasse übernommen. Mit manchen privaten Kassen kann sie (je nach Fläche) zum Teil analog zur Lasertherapie (GOÄ $24402885,2886)$ abgerechnet werden.

Das einfache Anwendungsprinzip macht die Behandlungsmethode gut an entsprechend geschultes Personal delegierbar. Dies macht prinzipiell einen sehr flexiblen Einsatz, vor allem im ambulanten Bereich, denkbar.

Im Jahr 2020 wurde ein „Plasmapatch“ derFirma Coldplasmatech zugelassen (Medizinprodukt Ilb), das mit einem selbstklebenden Applikator für eine flächige Anwendung bis zu $100 \mathrm{~cm}$ fungiert. Dieses ist als Wundauflage klassifiziert und darüber rezeptierbar - laut Hersteller auch nach neuem HHVG.

Im Juli 2021 wurde zudem vom G-BA dem Antrag auf Erprobung der Kaltplasmabehandlung bei chronischen Wunden stattgegeben. Im Erfolgsfall ist somit die Aufnahme in den Leistungskatalog der GKV zu erwarten.

\section{Schlussfolgerung}

Auch hier fehlen Level-1-Studien. Die aktuelle Datenlage zeigt auf, dass hier noch viele Fragen offen sind. Langfristige Ergebnisse - auch bezüglich potenzieller Nebenwirkungen - stehen noch aus.

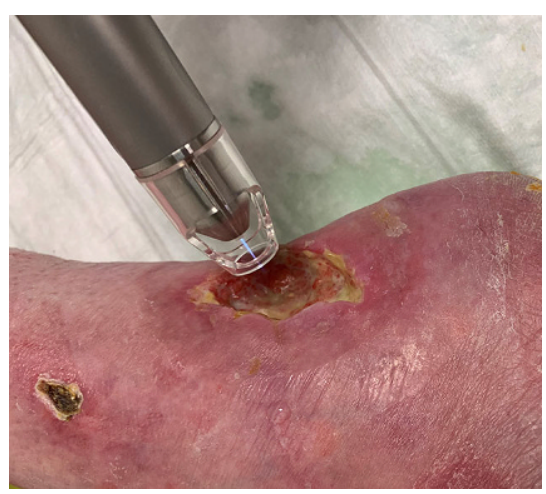

Abb. 5 ॥ Kaltplasma: Anwendung an einem Ulcus cruris arteriosum, Malleolus lateralis

\section{Laser (photobiomodulierende Therapie, PBM)}

\section{Wirkprinzip}

LASER steht für "Light Amplification by Stimulated Emission of Radiation“. Unter Laser versteht man sowohl den Generator als auch den Laserstrahl selbst. Er besteht schlussendlich aus elektromagnetischen Wellen hoher Intensität, die aufgrund ihrer Gleichgerichtetheit präzise gebündelt werden können Im Bereich der Wundversorgung kommen eine Vielzahl unterschiedlichster Laser als auch LEDs zum Einsatz. Seit 2014 werden alle biomedizinischen Applikationen von Licht unter dem Wort "Photobiomodulation“ subsummiert [21].

Es kommt zu einer Licht-Gewebe-Interaktion. Wirksam sind die Anteile, die im Gewebe eine Absorption oder Streuung erfahren. Hier spielt die gewählte Wellenlänge eine große Rolle. Wellenlängen von 450-1000 nm kommen in der Wundtherapie zum Einsatz. Neben der Lichtquelle oder Therapiedauer ist die Wirkung jedoch auch vom Gewebe selbst abhängig (z.B. durch Absorption geringe Eindringtiefe bei hohem Melaningehalt).

Je nach Lasertyp werden unterschiedliche Wellenlängen emittiert. So emittiert der Erbium:YAG-Laser z.B. Wellenlängen von $2940 \mathrm{~nm}$ oder der Rubinlaser $694 \mathrm{~nm}$ [21].

Es kommt zur direkten Übertragung von Lichtenergie auf molekularer Ebene. Durch Absorption von Licht werden Membranrezeptoren, Enzyme oder Mediatoren beeinflusst, wodurch es zu einer analgetischen 
und antiinflammatorischen Wirkung sowie einem erhöhten Angebot an Energieträgern kommt. Gleichzeitig werden reaktive Sauerstoffspezies gebildet, die die Proteinsynthese und Zellzykluskontrolle beeinflussen (z.B. Aktivierung des „transforming growth factor $\left.\beta^{\prime \prime}\right)$. Da dieser seinerseits viele Zellen im Wundmilieu, aber auch die Hämostase oder inflammatorische Zellen beeinflusst, gilt dies als der Haupteffekt im Bereich der Wundheilung. Es kommt zudem zu epithelialer Migration und Proliferation sowie Angiogenese [21].

\section{Wirksamkeit/Evidenz}

In Tierexperimenten konnte eine signifikante Verbesserung der Wundheilung durch photobiomodulierende Verfahren nachgewiesen werden [23].

Im Jahr 2019 veröffentlichten Mosca et al. ein Review, in das 11 Studien eingeschlossen werden konnten. Eine Reduktion des Schmerzempfindens sowie Juckreizes, aber auch eine verbesserte Wundheilung wurden beschrieben [21].

Ein systematisches Review von Brandao et al. et al. (2020), in welches 6 Studien eingeschlossen werden konnten, zeigte eine signifikante Verbesserung (Wundgröße, Granulationsgewebe, Schmerzen) der Wundheilung bei diabetischem Fußsyndrom (eingesetzte Wellenlängen: 632-685 nm) [5].

Petz et al. (2020) sowie Taradaj et al. (2013) evaluierten die Behandlung von Dekubiti. Für eine Wellenlänge von $658 \mathrm{~nm}$ (nicht jedoch für Wellenlängen von 808-840 nm) konnten signifikant erhöhte Abheilungsraten gezeigt werden [25, 28].

Kuffler (2016) empfahl in seinem Review für oberflächliche Wunden Wellenlängen zwischen $500-700 \mathrm{~nm}$ (tiefer liegende Läsionen: 800-1000 nm). Wellenlängen um $830 \mathrm{~nm}$ unterstützen zudem die Neoangiogenese. Im Endeffekt schlussfolgern die Kollegen, dass eine Kombination mehrerer Wellenlängen (simultan appliziert) den besten Effekt verspricht [15].

Alle Autoren zeigen sich kritisch aufgrund der fehlenden Vergleichbarkeit der Arbeiten $u$.a. bezüglich der applizierten Wellenlänge(n), sodass sie weitere Studien empfehlen, um die Wirksamkeit sinnvoll evaluieren zu können.

\section{Wirtschaftlichkeit}

Die Kosten der Behandlung werden von der Krankenkasse im Normalfall nicht übernommen. Mit manchen privaten Kassen kann sie wie die Kaltplasma-Applikation auch zum Teil analog zur dermatologischen Lasertherapie abgerechnet werden.

Prinzipiell birgt sie Potenzial einer deutlichen Kostenreduktion durch die Option der ambulanten Behandlung und Delegierbarkeit. Aktuell ist jedoch keine abschließende Beurteilung diesbezüglich möglich -, unter anderem da der Einsatz in Deutschland noch nicht weitverbreitet ist.

\section{Schlussfolgerung}

Photobiomodulierende Therapien zeigen vielversprechende Erfolge insbesondere bei der Behandlung des diabetischen Fußsyndroms sowie von Dekubiti. Die ausstehende Evaluation der optimalen Wellenlängen beziehungsweise die Heterogenität derselben in den aktuell vorliegenden Studien ermöglicht jedoch keine abschließende Beurteilung.

\section{Fazit für die Praxis}

- Alternative oder additive Methoden können die Notwendigkeit eines chirurgischen Debridements reduzieren.

- Nach einem primären chirurgischen Debridement oder für stagnierende Wunden, die durch das Vorhandensein avitalen Gewebes an der Heilung gehindert werden, können die hier vorgestellten Methoden einen Benefit bieten.

- Eine Stärke der physikalischen Interventionen liegt in der potenziellen Reduktion der Keimlast auch multiresistenter Erreger.

- Durch den Einsatz additiver physikalischer Methoden können Patienten teilweise (frühzeitig) ambulant ohne Narkosenotwendigkeit behandelt und weitere operative Wundrevisionen vermieden werden.

- Die dadurch mögliche Kostenersparnis sowie die Vermeidung oder Verkürzung eines Krankenhausaufenthaltes sind als Vorteile anzusehen.

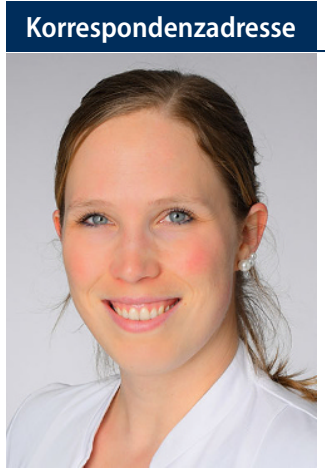

\section{Dr. Ursula Elisabeth Maria Werra}

Klinik für Gefäßchirurgie, Vaskuläre und endovaskuläre Chirurgie, Uniklinik Köln Kerpener Str. 62, 50937 Köln, Deutschland ursula.werra@uk-koeln.de

Funding. Open Access funding enabled and organized by Projekt DEAL.

\section{Einhaltung ethischer Richtlinien}

Interessenkonflikt. U.E.M. Werra, M. Zinser und B. Dorweiler geben an, dass kein Interessenkonflikt besteht.

Für diesen Beitrag wurden von den Autoren keine Studien an Menschen oder Tieren durchgeführt. Für die aufgeführten Studien gelten die jeweils dort angegebenen ethischen Richtlinien.

Open Access. Dieser Artikel wird unter der Creative Commons Namensnennung 4.0 International Lizenz veröffentlicht, welche die Nutzung, Vervielfältigung, Bearbeitung, Verbreitung und Wiedergabe in jeglichem Medium und Format erlaubt, sofern Sie den/die ursprünglichen Autor(en) und die Quelle ordnungsgemäß nennen, einen Link zur Creative Commons Lizenz beifügen und angeben, ob Änderungen vorgenommen wurden.

Die in diesem Artikel enthaltenen Bilder und sonstiges Drittmaterial unterliegen ebenfalls der genannten Creative Commons Lizenz, sofern sich aus der Abbildungslegende nichts anderes ergibt. Sofern das betreffende Material nicht unter der genannten Creative Commons Lizenz steht und die betreffende Handlung nicht nach gesetzlichen Vorschriften erlaubt ist, ist für die oben aufgeführten Weiterverwendungen des Materials die Einwilligung des jeweiligen Rechteinhabers einzuholen.

Weitere Details zur Lizenz entnehmen Sie bitte der Lizenzinformation auf http://creativecommons.org/ licenses/by/4.0/deed.de.

\section{Literatur}

1. https://www.aerzteblatt.de/nachrichten/66620/ Versorgung-von-Wunderkrankungen-kostetacht-Milliarden-Euro. Zugegriffen: 6. Juni 2021

2. Amini S, ShojaeeFard A (2013) Low-frequency ultrasound debridement in patients with dia- 
betic foot ulcers and osteomyelitis. Wounds 25(7):193-198

3. Assadian O, Ousey KJ (2019) Effects and safety of atmospheric low-temperature plasma on bacterial reduction in chronic wounds and wound size reduction: A systematic review and meta-analysis. Int Wound J 16:103-111

4. AWMF Leitlinie (2012) Lokaltherapie chronischer Wunden bei Patienten mit den Risiken periphere arterielle Verschlusskrankheit, Diabetes mellitus, chronisch venöse Insuffizienz

5. Brandão MGSA, Ximenes MAM (2020) Effects of low-level laser therapy on the healing of foot ulcers in people with diabetes mellitus. Estima Braz J Enteros Ther 18:e320

6. Brehmer F, Haenssle HA (2015) Alleviation of chronic venous leg ulcers with a hand-held dielectric barrier discharge plasma generator (PlasmaDerm $\left({ }^{\circledR}\right)$ VU-2010): results of a monocentric, two-armed, open, prospective, randomized and controlled trial (NCT01415622). J Eur Acad Dermatol Venereol 29(1):148-155

7. Chang YR, PerryJ (2017) Low-frequencyultrasound debridement in chronic wound healing: a systematic review of current evidence. Plast Surg (Oakv) 25(1):21-26

8. Cullum N, Liu Z (2017) Therapeutic ultrasound for venous leg ulcers. Cochrane Database Syst Rev 5(5):CD1180

9. Dissemond J, Augustin M (2014) Moderne Wundtherapie - praktische Aspekte der lokalen, nichtinterventionellen Behandlung von Patienten mit chronischen Wunden. J Dtsch Dermatol Ges 12:541-554

10. Goepel L, Heyer K (2015) Selektivverträge zu chronischen Wunden - aktueller Stand in Deutschland. Gefässchirurgie 20:18-24

11. Haertel B, von Woedtke T (2014) Non-thermal atmospheric-pressure plasma possible application in wound healing. Biomol Ther (Seoul) 22(6):477-490

12. Herberger $K$, Franzke N (2011) Efficacy, tolerability and patient benefit of ultrasound-assisted wound treatment versus surgical debridement: a randomized clinical study. Dermatology 222(3):244-249

13. Isbary G, Morfill G (2010) A first prospective randomized controlled trial to decrease bacterial load using cold atmospheric argon plasma on chronic wounds in patients. $\mathrm{Br} J$ Dermatol 163(1):78-82

14. Isbary G, Heinlin J (2012) Successful and safe use of 2 min cold atmospheric argon plasma in chronic wounds: results of a randomized controlled trial. $\mathrm{Br}$ JDermatol 167:404-410

15. Kuffler DP (2016) Photobiomodulation in promoting wound healing: a review. Regen Med 11(1):107-122

16. Lázaro-Martínez JL, Álvaro-Afonso FJ (2018) Ultrasound-assisted debridement of neuroischaemic diabetic foot ulcers, clinical and microbiological effects: a case series.J Wound Care 27(5):278-286

17. Lázaro-Martínez JL, Álvaro-Afonso FJ (2020) Cellularproliferation, dermal repair, and microbiological effectiveness of ultrasound-assisted wound debridement (UAW) versus standard wound treatment in complicated diabetic foot ulcers (DFU): an open-label randomized controlled trial. J Clin Med 9(12):4032

18. Gan L, Jiang J (2021) Cold atmospheric plasma applications in dermatology: a systematic review. JBiophotonics 14:e202000415

19. LuTheryn G, Glynne-Jones P (2020) Ultrasoundmediated therapies for the treatment of biofilms in

\section{Physical interventions as an alternative/addition to surgical debridement. Ultrasound, coldplasma and LASER/photobiomodulation}

Background: In Germany the treatment of patients with chronic wounds costs 8 billion Euros annually.

Often, surgical debridement is indicated. But if critical structures are directly adjacent or the patient has only a limited ability to undergo anaesthesia, the possible applications can be limited.

Furthermore, for financial reasons, the possibility of delegation is always of interest. Objectives: We present alternative and additional methods to surgical debridement from the portfolio of physical interventions.

Materials and methods: Ultrasound-assisted wound debridement (UAW), cold plasma and photobiomodulation are based on interesting principles with great potential for development. The respective mechanisms, the current evidence, as well as financial aspects, are presented.

Results: Overall, mainly because of the heterogeneity of the data the current evidence is weak. Also, some aspects of the treatment modalities have not yet been fully clarified. UAW represents a real alternative. It is highly acceptable to patients and can be performed in an outpatient setting.

The effectiveness of cold plasma or photobiomodulation still cannot be assessed conclusively. However, several positive clinical results have been shown.

Conclusions: Surgical debridement as such (still) cannot be completely replaced. However, the alternative methods such as UAW can offer an additional benefit. Wounds may be treated in an outpatient setting earlier (with corresponding savings). Another important strength is moreover the effectiveness regarding multidrug-resistant germs. There is a need for further clinical studies to be initiated, as the current data show that there are still many unanswered questions.

\section{Keywords}

Modern wound therapy · Photobiomodulation · Ultrasound-assisted debridement · Plasma . Chronic wounds

chronic wounds: a review of present knowledge. Microb Biotechnol 13(3):613-628

20. Moelleken $M$, JockenhöferF (2020) Pilotstudie zum Einfluss von kaltem atmosphärischem Plasma auf bakterielle Kontamination und Heilungstendenz chronischer Wunden. J Dtsch Dermatol Ges 18(10):1094-1102

21. Mosca RC, Adrian A (2019) Photobiomodulation therapy for wound care: a potent, noninvasive, photoceutical approach. Adv Ski Wound Care 32(4):157-167

22. Nationale Versorgungsleitlinie (2009) Typ-2-Diabetes: Präventions- und Behandlungsstrategien fürFußkomplikationen

23. Peplow PV, Chung TY (2010) Laser photobiomodulation of wound healing: a review of experimental studiesin mouseand ratanimal models. Photomed Laser Surg 28(3):291-325

24. Bundesärztekammerund Kassenärztliche Bundesvereinigung (2008) Persönliche Leistungserbringung-Möglichkeiten und Grenzen derDelegation ärztlicher Leistungen (Stand: 29.08.2008)

25. Petz Fd FC, Félix JVC (2020) Effect of photobiomodulation on repairing pressure ulcers in adult and elderly patients: a systematic review. Photochem Photobiol 96:191-199

26. Stratmann B, Costea TC (2020) Effect of cold atmospheric plasma therapy vs standard therapy placebo on wound healing in patients with diabetic foot ulcers: a randomized clinical trial. JAMA Netw Open 3(7):e2010411
27. Swanson T, Lázaro-Martínez JL (2020) Ultrasonicassisted wound debridement: report from a closed panel meeting. JWound Care 29(2):128-135

28. Taradaj J, Halski T (2013) Effect of laser irradiation at different wavelengths $(940,808$, and $658 \mathrm{~nm})$ on pressure ulcer healing: results from a clinical study. Evid Based Complement Alternat Med 2013:960240

29. Voigt J, Wendelke M (2011) Low-frequency ultrasound $(20-40 \mathrm{kHz})$ as an adjunctive therapy for chronic wound healing: A systematic review of the literature and meta-analysis of eight randomized controlled trials. Int J Extrem Wound 10(4):190-199

30. Zoremba N,Coburn M(2019) Delirim Krankenhaus. Dtsch Arztebl Int 116:101-106 